\title{
二维碳化钛/碳纳米管负载铂钉粒子的制备及电催化性能研究
}

\author{
李亚辉 ${ }^{1}$, 张建峰 ${ }^{1}$, 曹惠杨 ${ }^{1}$, 张 欣 ${ }^{1}$, 江 莞 ${ }^{1,2}$ \\ (1. 河海大学 力学与材料学院, 南京 $211100 ; 2$. 东华大学 材料科学与工程学院, 上海 201620)
}

摘 要: 直接甲醇燃料电池因操作方便、转化效率高、操作温度低、污染少以及液体燃料易存储易运输等优势具有良 好的应用前景, 但现有阳极催化剂存在催化活性低、抗 CO 中毒性差等缺点, 制约了其商业化应用前景。本研究采用 三步法制备得到了一系列不同 $\mathrm{Pt} 、 \mathrm{Ru}$ 配比的 $\mathrm{PtRu} /\left(\mathrm{Ti}_{3} \mathrm{C}_{2} \mathrm{~T}_{x}\right)_{0.5}$-(MWCNTs $)_{0.5}$ 阳极催化剂材料, $\mathrm{HF}$ 腐蚀 $\mathrm{Ti}_{3} \mathrm{AlC}_{2}$ 得到 $\mathrm{Ti}_{3} \mathrm{C}_{2} \mathrm{~T}_{x}$ ，与酸化处理的多壁碳纳米管(MWCNTs)复合后通过溶剂热法负载 Pt、Ru 颗粒。通过 XRD、SEM、EDS、TEM、 XPS 等分析铂钓的协同关系。结果表明: Ru 原子与 Pt 原子晶格混合, 形成了粒径约 $3.6 \mathrm{~nm}$ 的铂钉双金属合金。电化 学分析结果表明: $\mathrm{Pt}_{1} \mathrm{Ru}_{0.5} /\left(\mathrm{Ti}_{3} \mathrm{C}_{2} \mathrm{~T}_{x}\right)_{0.5}$-(MWCNTs $)_{0.5}$ 催化剂具有最佳的电化学性能, 其电化学活性面积(Electrochemical Active Area, ECSA)为 $139.5 \mathrm{~m}^{2} / \mathrm{g}$, 正向峰电流密度为 $36.4 \mathrm{~mA} / \mathrm{cm}^{2}$ 。

关 键 词: 二维 $\mathrm{Ti}_{3} \mathrm{C}_{2} \mathrm{~T}_{x}$ 材料; 铂钉纳米粒子; 直接甲醇燃料电池; 电催化性能

中图分类号: TQ174 文献标识码: A

\section{PtRu Particles Supported on Two-dimensional Titanium Carbide/Carbon Nanotubes: Preparation and Electrocatalytic Properties}

\author{
LI Ya-Hui ${ }^{1}$, ZHANG Jian-Feng ${ }^{1}$, CAO Hui-Yang ${ }^{1}$, ZHANG Xin ${ }^{1}$, JIANG Wan ${ }^{1,2}$ \\ (1. College of Mechanics and Materials, Hohai University, Nanjing 211100, China; 2. College of Materials Science and Engi- \\ neering, Donghua University, Shanghai 210050, China)
}

\begin{abstract}
Direct methanol fuel cells have good application prospects due to their advantages of convenient operation, high conversion efficiency, low operating temperature, low pollution, and easy storage and easy transportation of liquid fuel. However, existing anode catalysts have shortcomings such as low catalytic activity and poor resistance to $\mathrm{CO}$ toxicity which restrict its commercial application. In this study, a series of PtRu/ $\left(\mathrm{Ti}_{3} \mathrm{C}_{2} \mathrm{~T}_{x}\right)_{0.5}$-(MWCNTs $)_{0.5}$ anode catalyst materials with different $\mathrm{Pt}$ and Ru ratios were prepared by three-step method. $\mathrm{Ti}_{3} \mathrm{C}_{2} \mathrm{~T}_{x}$ was obtained by $\mathrm{HF}$ corrosion of $\mathrm{Ti}_{3} \mathrm{AlC}_{2}$, and acidified multi-walled carbon nanotubes (MWCNTs). After the compounding, Pt and Ru particles are supported by a solvothermal method. The synergistic relationship of Ru and Pt atoms was analyzed by XRD, SEM, EDS, TEM, and XPS. The results show that the Ru atoms are mixed with the Pt atoms to form PtRu bimetallic alloy with a particle size of about $3.6 \mathrm{~nm}$. The electrochemical results show that the $\mathrm{Pt}_{1} \mathrm{Ru}_{0.5} /\left(\mathrm{Ti}_{3} \mathrm{C}_{2} \mathrm{~T}_{x}\right)_{0.5}-(\mathrm{MWCNTs})_{0.5}$ catalyst has the best electrochemical performance. Its electrochemical active area (ECSA) is $139.5 \mathrm{~m}^{2} / \mathrm{g}$, and positive peak current density is $36.4 \mathrm{~mA} / \mathrm{cm}^{2}$.
\end{abstract}

Key words: two-dimensional $\mathrm{Ti}_{3} \mathrm{C}_{2} \mathrm{~T}_{x}$ material; PtRu nanoparticle; direct methanol fuel cells; electrocatalytic performance

收稿日期: 2019-03-12; 收到修改稿日期：2019-06-25

基金项目：国家重点研发计划(2018YFC1508704); 江苏省自然科学基金(BK20161506); 国家自然科学基金(51432004) National Key R\&D Program of China (2018YFC1508704); Jiangsu Provincial Natural Science Foundation of China (BK20161506); National Natural Science Foundation of China (51432004)

作者简介: 李亚辉(1994-), 男, 硕士研究生. E-mail: 15951935290@163.com

LI Ya-Hui (1994-), male, Master candidate. E-mail: 15951935290@163.com

通讯作者: 张建峰, 教授. E-mail: jfzhang_sic@163.com

ZHANG Jian-Feng, professor. E-mail: jfzhang_sic@163.com 
工业迅速发展引起的化石燃料的急剧消耗, 迫 使人们不断开发新型能源技术 ${ }^{[1]}$, 直接甲醇燃料电 池 (DMFC) 以其能量转化率高、污染少、安全可靠 和电化学活性高等优势成为能源领域的研究热点之 - [2-3]。DMFC 阳极催化剂主要为 $\mathrm{Pt}$ 基催化剂, $\mathrm{Pt}$ 金属抗 $\mathrm{CO}$ 中毒能力差和稳定性差等缺点, 制约着 $\mathrm{DMFC}$ 的发展 ${ }^{[4-5]}$, 而合成以 $\mathrm{PtRu}$ 为代表的二元金 属催化剂是解决这一问题的有效策略 ${ }^{[6-7]}$ 。与一元 $\mathrm{Pt}$ 催化剂相比, 二元 PtRu 催化剂的电催化性能的机 理更为复杂 ${ }^{[8]}$ 。催化剂中 $\mathrm{Pt} 、 \mathrm{Ru}$ 的原子比例及 $\mathrm{Ru}$ 的存在形式均会对催化性能产生影响。因此, 选择 合适的 Pt、Ru 的原子比, 制备合适粒径、分散均匀 的 PtRu 纳米粒子, 对提高 DMFC 阳极催化剂的性 能具有重要意义。

二维材料 MXenes ${ }^{[9-10]}$ 具有大的比表面积, 表面 存在的断键可以提供更大的有效活性面积, 有利于 贵金属催化剂的均匀负载。目前, 二维材料 MXenes 已逐渐在储能 ${ }^{[11-13]}$ 、吸波 ${ }^{[14]}$ 、催化 ${ }^{[15-16]}$ 、传感器 ${ }^{[17]}$ 、 储氢 ${ }^{[18]}$ 等诸多领域展现出巨大的应用价值。自 Naguib 和 Barsoum 等 ${ }^{[19]}$ 利用氢氟酸刻蚀 $\mathrm{Ti}_{3} \mathrm{AlC}_{2}$ 中 的 $\mathrm{Al}$ 原子层, 制得 $\mathrm{Ti}_{3} \mathrm{C}_{2} \mathrm{~T}_{x}$ 以来, 因其特殊的表面 结构被认为是极具应用前景的电极材料。2014 年, $\mathrm{Xie}$ 等 ${ }^{[20]}$ 制备了 $\mathrm{Pt} / \mathrm{Ti}_{3} \mathrm{C}_{2} \mathrm{~T}_{x}$ 一元金属催化剂, 其电催 化活性、稳定性均优于商用的 $\mathrm{Pt} / \mathrm{C}$ 催化剂。另外, 碳 材料一直是 Pt 基催化剂载体的研究热点, 比如炭 黑 ${ }^{[21]}$ 、碳纤维 ${ }^{[22]}$ 、多孔碳 ${ }^{[23]}$ 、碳纳米管 ${ }^{[24]}$ 和石墨烯 ${ }^{[25]}$ 等。一维碳纳米管具有独特的中空无缝管状结构, 管壁是六元环结构, 广泛应用于储氢、催化和复合 材料增强体等领域。经过酸化处理后的碳纳米管表 面带有较多的含氧官能团, 与 $\mathrm{Ti}_{3} \mathrm{C}_{2} \mathrm{~T}_{x}$ 复合后, 两者 表面相互作用, 交联成特殊的三维结构, 这一结构 可以为催化剂粒子的负载提供更多的针定位点。

本研究以 $m\left(\mathrm{Ti}_{3} \mathrm{C}_{2} \mathrm{~T}_{x}\right): m\left(\mathrm{Ti}_{3} \mathrm{C}_{2} \mathrm{~T}_{x}: \mathrm{MWCNTs}\right)=$ $1: 1$ 的复合材料为载体, 通过溶剂热法, 合成均匀 分散、粒径较小的 $\mathrm{PtRu} /\left(\mathrm{Ti}_{3} \mathrm{C}_{2} \mathrm{~T}_{x}\right)_{0.5}$-(MWCNTs $)_{0.5}$ 催 化剂。以乙二醇为溶剂, 同时还原金属 $\mathrm{Pt} 、 \mathrm{Ru}$ 和部 分含氧官能团, 通过优化电催化剂的制备工艺, 获 得了高活性 $\mathrm{PtRu} /\left(\mathrm{Ti}_{3} \mathrm{C}_{2} \mathrm{~T}_{x}\right)_{0.5}$-(MWCNTs $)_{0.5}$ 催化剂, 极大提高了催化剂的稳定性。研究了 $\mathrm{Pt} 、 \mathrm{Ru}$ 原子比 对催化剂活性和稳定性的影响规律, 分析了反应机 理, 为 DMFC 催化剂实用化的进程奠定了基础。

\section{1 实验方法}

\section{1 试剂和材料}

实验用 $\mathrm{Ti}_{3} \mathrm{AlC}_{2}$ 粉末购自福曼斯科技(北京)有限
公司 (粒径 $<38 \mu \mathrm{m})$; 羟基化碳纳米管粉末购自中科 院成都有机化学有限公司(长度: 10 30 $\mu \mathrm{m}$ )。实验所 用试剂 $\mathrm{K}_{2} \mathrm{PdCl}_{4}$ 溶液(Alfa Aesar，中国) $\mathrm{RuCl}_{3}$ 溶液、 Nafion 溶液(Dupont, 美国)、氢氟酸 $(\mathrm{HF}, 40 \mathrm{wt} \%$ )、无 水乙醇 $\left(\mathrm{C}_{2} \mathrm{H}_{5} \mathrm{OH}\right)$ 、乙二醇 $\left(\left(\mathrm{CH}_{2} \mathrm{OH}\right)_{2}\right)$ 、甲醇 $\left(\mathrm{CH}_{3} \mathrm{OH}\right)$ 、 硫酸 $\left(\mathrm{H}_{2} \mathrm{SO}_{4}\right)$ 均为分析纯级别(Analytical grade, AR)。 $\mathrm{Ti}_{3} \mathrm{AlC}_{2}$ 在 $25{ }^{\circ} \mathrm{C}$ 下经 $\mathrm{HF}$ 液相刻蚀 $48 \mathrm{~h}$ 后获得 $\mathrm{Ti}_{3} \mathrm{C}_{2} \mathrm{~T}_{x}$ 粉末。碳纳米管在混酸溶液 $\left(V\left(\mathrm{H}_{2} \mathrm{SO}_{4}\right)\right.$ : $\left.V\left(\mathrm{HNO}_{3}\right)=3: 1\right)$ 下酸化得到酸化碳纳米管(MWCNTs)。

\section{$1.2 \mathrm{PtRu} /\left(\mathrm{Ti}_{3} \mathrm{C}_{2} \mathrm{~T}_{x}\right)_{0.5}-(\mathrm{MWCNTs})_{0.5}$ 的制备}

以 $m\left(\mathrm{Ti}_{3} \mathrm{C}_{2} \mathrm{~T}_{x}\right): m\left(\mathrm{Ti}_{3} \mathrm{C}_{2} \mathrm{~T}_{x}: \mathrm{MWCNTs}\right)=1: 1$ 的 复合材料为载体, 通过溶剂热法, 合成均匀分散、较 小粒径的 $\mathrm{PtRu} /\left(\mathrm{Ti}_{3} \mathrm{C}_{2} \mathrm{~T}_{x}\right)_{0.5}$-(MWCNTs $)_{0.5}$ 催化剂, 合 成过程如图 1 所示。称取 $10 \mathrm{mg} \mathrm{Ti}_{3} \mathrm{C}_{2} \mathrm{~T}_{x}$ 和 $10 \mathrm{mg}$ MWCNTs 置于烧杯中, 加入 $40 \mathrm{~mL}$ 水和 $40 \mathrm{~mL}$ 乙二 醇, 超声 $1 \mathrm{~h}$ 。之后加入 $256 \mu \mathrm{L} 0.1 \mathrm{~mol} / \mathrm{L} \mathrm{K}_{2} \mathrm{PtCl}_{4}$ 和 $64 \mu \mathrm{L} 0.1 \mathrm{~mol} / \mathrm{L} \mathrm{RuCl}_{3}$ 溶液, 搅拌 $15 \mathrm{~min}$ 后倒于聚 四氟乙烯内祄不锈钢反应釜中, $120{ }^{\circ} \mathrm{C}$ 下反应 $18 \mathrm{~h}$ 。 多次离心水洗产物, 再置于冷冻干燥机内冻干。调 节 $\mathrm{Pt}$ 和 $\mathrm{Ru}$ 的摩尔比, 如 $1: 0.25 、 1: 0.5 、 1: 1$ 和 $1: 2$, 分别记为 $\mathrm{Pt}_{1} \mathrm{Ru}_{0.25} / \mathrm{TM} 、 \mathrm{Pt}_{1} \mathrm{Ru}_{0.5} / \mathrm{TM} 、 \mathrm{Pt}_{1} \mathrm{Ru}_{1} / \mathrm{TM}$ 和

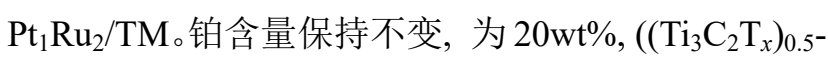
$(\mathrm{MWNTs})_{0.5}$ 简写为 TM)。

\section{3 材料表征}

采用 $\mathrm{X}$ 射线粉末衍射仪 (XRD, Bruker D8 Advance $X)$ 测定样品的物相组成, 采用场发射扫描电 子显微镜(SEM, HITACHI S-4800)、透射电镜(TEM, Tecnai G2 F30 S-TWIN)观察样品微观形貌, 采用光 电子能谱仪(XPS, ESCALAB 250Xi) 分析样品组成。

\section{4 电极制备及电化学性能测试}

采用 $\mathrm{CHI} 760 \mathrm{E}$ 电化学工作站三电极体系测试催 化剂的电化学性能, 对电极是直径 $0.5 \mathrm{~mm}$ 的铂丝, 参比电极为饱和甘录电极, 以本实验制得的催化剂 修饰的玻碳电极为工作电极。电极体系在 $1 \mathrm{~mol} / \mathrm{L}$ $\mathrm{H}_{2} \mathrm{SO}_{4}$ 进行循环伏安测试时, 电势扫描范围为 $-0.2 \sim 1 \mathrm{~V}$, 扫描速率 $20 \mathrm{mV} / \mathrm{s}$; 在 $1 \mathrm{~mol} / \mathrm{L} \mathrm{H}_{2} \mathrm{SO}_{4}+$

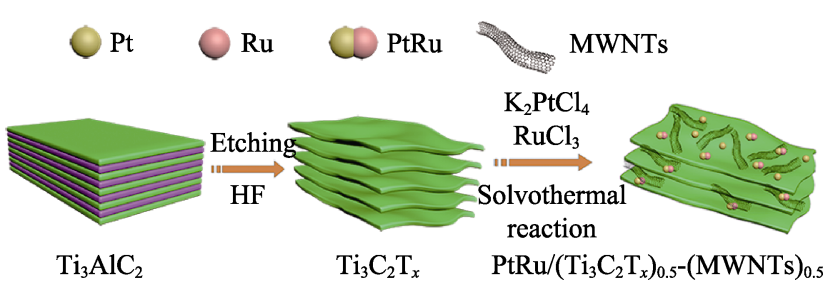

图 1 合成 $\mathrm{PtRu} /\left(\mathrm{Ti}_{3} \mathrm{C}_{2} \mathrm{~T}_{x}\right)_{0.5}-(\mathrm{MWCNTs})_{0.5}$ 过程示意图

Fig. 1 Schematic preparation process for $\mathrm{PtRu} /\left(\mathrm{Ti}_{3} \mathrm{C}_{2} \mathrm{~T}_{x}\right)_{0.5^{-}}$ (MWCNTs) $)_{0.5}$ catalysts 
$2 \mathrm{~mol} / \mathrm{L} \mathrm{CH}_{3} \mathrm{OH}$ 测试时，电压扫描范围为 $0 \sim 1 \mathrm{~V}$, 扫 描速率 $20 \mathrm{mV} / \mathrm{s}$ 。电化学交流阻抗法(EIS)是在 $1 \mathrm{~mol} / \mathrm{L}$ $\mathrm{H}_{2} \mathrm{SO}_{4}+2 \mathrm{~mol} / \mathrm{L} \mathrm{CH} \mathrm{CH}_{3} \mathrm{OH}$ 混合溶液中测试, 初始电 压为开路电位, 低频 $0.01 \mathrm{~Hz}$, 高频 $10^{5} \mathrm{~Hz}$; 计时电 流法(IT) 是在 $1 \mathrm{~mol} / \mathrm{L} \mathrm{H}_{2} \mathrm{SO}_{4}+2 \mathrm{~mol} / \mathrm{L} \mathrm{CH}_{3} \mathrm{OH}$ 混合 溶液中测试, 运行时间 $3000 \mathrm{~s}$, 恒定电压为 $0.5 \mathrm{~V}$ 。

\section{2 结果与讨论}

\section{1 结构表征}

图 2(a)是不同 $\mathrm{Ru}$ 掺量的 $\mathrm{PtRu} / \mathrm{TM}$ 复合材料的 $\mathrm{XRD}$ 图谱。所有曲线均可以清晰地看到各个组分的 特征峰, 证实 $\mathrm{Pt} 、 \mathrm{Ti}_{3} \mathrm{C}_{2} \mathrm{~T}_{x}$ 和 MWCNTs 复合在一起。 在 $2 \theta \approx 38.94^{\circ} 、 45.12^{\circ} 、 67.52^{\circ}$ 和 $81.02^{\circ}$ 处的衍射峰 分别对应于 Pt 的(111)、(200)、(220)和(311)晶面, 这 与 $\mathrm{Pt}$ 的标准峰相一致 ${ }^{[26]}$, 即形成了面心立方晶系的 $\mathrm{Pt}$ 颗粒(fcc), 有金属态铂存在。图谱中未出现钉金属 或钉氧化物的特征衍射峰, 说明 Pt、Ru 双金属发生 合金化 ${ }^{[27]}$ ，有利于除去甲醇氧化生成的中间产物 ${ }^{[28]}$ 。

图 2(b)是 $\mathrm{Pt}_{1} \mathrm{Ru}_{0.5} / \mathrm{TM}$ 催化剂的 $\mathrm{SEM}$ 照片, 可 以观测到 $\mathrm{Pt}_{1} \mathrm{Ru}_{0.5} / \mathrm{TM}$ 具有典型的二维片层状 $\mathrm{Ti}_{3} \mathrm{C}_{2} \mathrm{~T}_{x}$ 结构及与管状 MWCNTs 交缠连接形成 的三维立体 结构, 这有利于为金属纳米粒子提供更大的载体表
面，便于金属纳米粒子的有效负载。MWCNTs 的引入 也有利于连接 $\mathrm{Ti}_{3} \mathrm{C}_{2} \mathrm{~T}_{x}$ 片层, 便于离子和电子的快速 输运，并在一定程度上减弱 $\mathrm{Ti}_{3} \mathrm{C}_{2} \mathrm{~T}_{x}$ 的团聚。图 2(c c d ) 为 $\mathrm{Pt}_{1} \mathrm{Ru}_{0.5} / \mathrm{TM}$ 的透射电镜照片, MWCNTs 与 $\mathrm{Ti}_{3} \mathrm{C}_{2} \mathrm{~T}_{x}$ 片层交叉缠结形成较大的载体, 球形的 PtRu 合金颗 粒均匀分布在载体表面。酸化处理后的 MWCNTs 表面含有羟基、羧基等含氧官能团，含氧官能团的 存在便于贵金属颗粒有效针定及均匀负载在载体 $\mathrm{Ti}_{3} \mathrm{C}_{2} \mathrm{~T}_{x}$-MWCNTs 上。通过粒径分析计算软件计算 出催化剂的粒径约为 $3.6 \mathrm{~nm}$ 。

图 3(a f)为 $\mathrm{Pt}_{1} \mathrm{Ru}_{0.5} / \mathrm{TM}$ 的 $\mathrm{EDS}$ 元素面扫图。 $\mathrm{Pt}$ 和 $\mathrm{Ru}$ 颗粒分散均匀, 含量较多, 且存在位置相同, 这与 XRD 分析的 PtRu 合金化结论一致, 说明金属 粒子被还原负载在载体 $\mathrm{Ti}_{3} \mathrm{C}_{2} \mathrm{~T}_{x}$-MWCNTs 上。

图 4(a) 是 $\mathrm{Pt} / \mathrm{TM}$ 和 $\mathrm{Pt}_{1} \mathrm{Ru}_{0.5} / \mathrm{TM}$ 的 XPS 全谱图。 与 $\mathrm{Pt} / \mathrm{TM}$ 相比, $\mathrm{Pt}_{1} \mathrm{Ru}_{0.5} / \mathrm{TM}$ 中多了 $\mathrm{Ru}$ 元素的信号峰, 表明 $\mathrm{Ru}$ 元素负载在表面上。图 4(b)是 $\mathrm{Pt} / \mathrm{TM}$ 和 $\mathrm{Pt}_{1} \mathrm{Ru}_{0.5} / \mathrm{TM}$ 的 $\mathrm{O} 1 \mathrm{~s}$ 分谱图。 $\mathrm{Ti}_{3} \mathrm{C}_{2} \mathrm{~T}_{x}$-MWCNTs 表面 的含氧官能团保证 Pt、Ru 合金粒子负载, 形成 $\mathrm{Pt} / \mathrm{TM}$ 和 $\mathrm{Pt}_{1} \mathrm{Ru}_{0.5} / \mathrm{TM}$ 复合催化剂。 $\mathrm{Pt}_{1} \mathrm{Ru}_{0.5} / \mathrm{TM}$ 催化 剂的 $\mathrm{Ti}-\mathrm{OH}$ 键含量和 $\mathrm{Pt} / \mathrm{TM}$ 相当, Ti-OH 键含量远 大于 $\mathrm{Ti}-\mathrm{O}$ 键时, 说明 $\mathrm{Ti}$ 表面带有大量的 $-\mathrm{OH}$, 削弱 了 $\mathrm{Ti}$ 原子的电子密度, 促进电子转移。图 $4(\mathrm{c})$ 是 $\mathrm{Pt} / \mathrm{TM}$
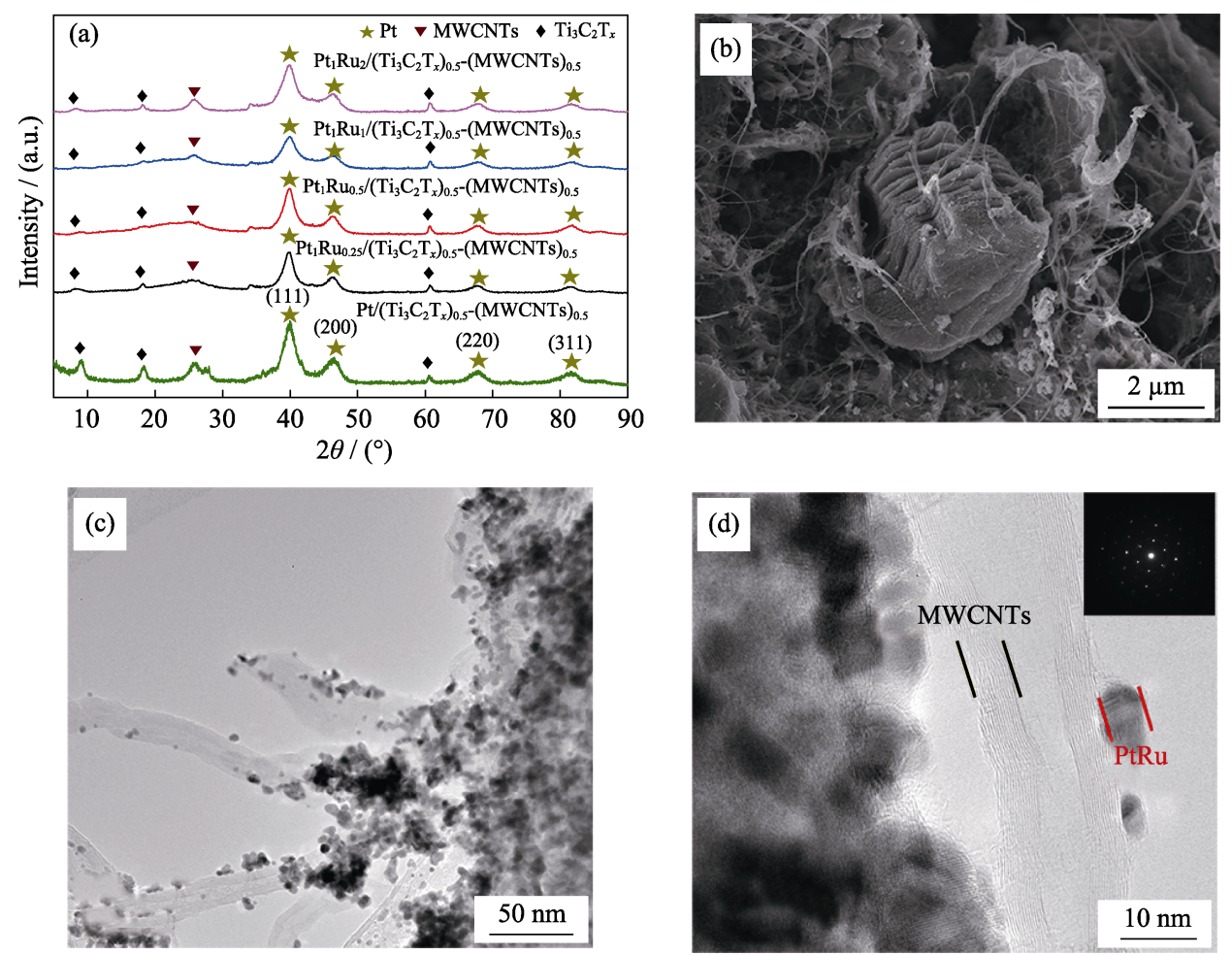

图 2 (a)不同 $\mathrm{Ru}$ 掺量的 $\mathrm{PtRu} / \mathrm{TM}$ 复合材料的 $\mathrm{XRD}$ 图谱、(b) $\mathrm{Pt}_{1} \mathrm{Ru}_{0.5} / \mathrm{TM}$ 催化剂的 $\mathrm{SEM}$ 照片和(c $\sim \mathrm{d}$ ) $\mathrm{Pt}_{1} \mathrm{Ru}_{0.5} / \mathrm{TM}$ 的 TEM 照片及选区电子衍射结果

Fig. 2 (a) XRD patterns of PtRu/TM with different Ru contents, (b) SEM images of $\mathrm{Pt}_{1} \mathrm{Ru}_{0.5} / \mathrm{TM}$; (c-d) HRTEM and selected area electron diffraction (SAED) pattern of $\mathrm{Pt}_{1} \mathrm{Ru}_{0.5} / \mathrm{TM}$ 

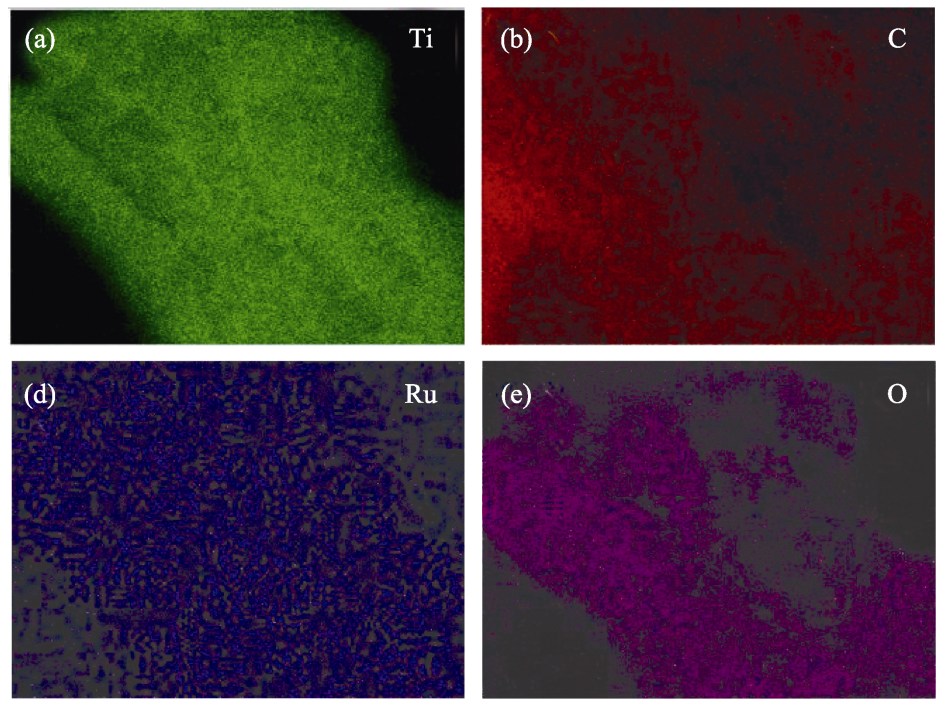

图 $3 \mathrm{Pt}_{1} \mathrm{Ru}_{0.5} / \mathrm{TM}$ 的 EDS 面扫图: (a) Ti, (b) C, (c) Pt, (d) Ru, (e) O; (f)相应的 SEM 照片

Fig. 3 (a) Ti, (b) C, and (c) Pt, (d) Ru, and (e) O, EDS images of $\mathrm{Pt}_{1} \mathrm{Ru}_{0.5} / \mathrm{TM}$, and (f) corresponding SEM image
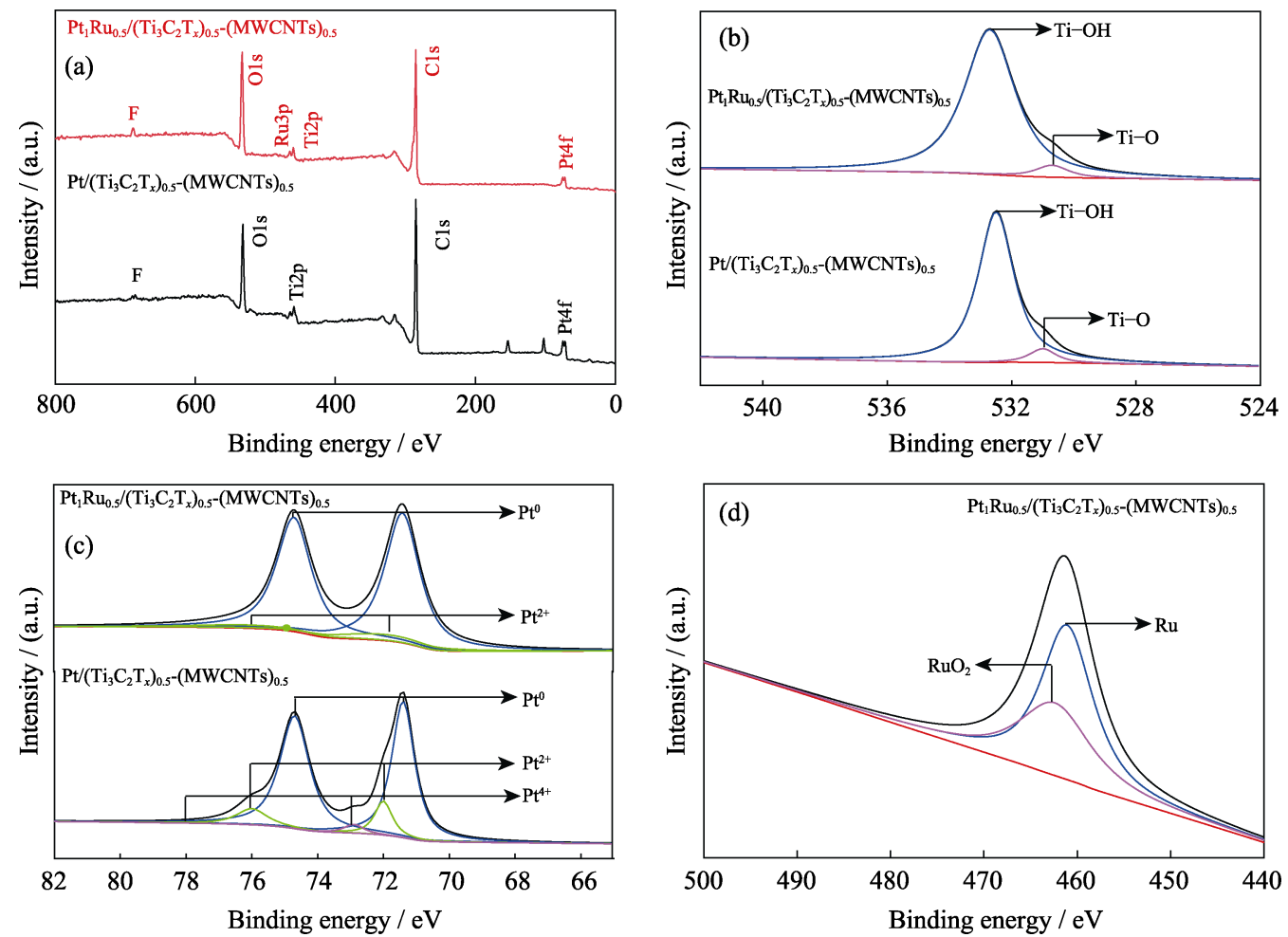

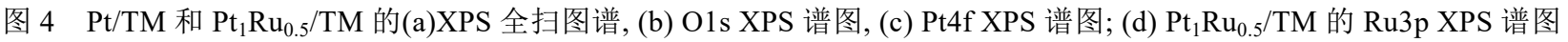
Fig. 4 (a) XPS survey spectra, (b) high-resolution O1s core-level XPS spectra, (c) high-resolution Pt4f core-level XPS spectra of $\mathrm{Pt} / \mathrm{TM}$ and $\mathrm{Pt}_{1} \mathrm{Ru}_{0.5} / \mathrm{TM}$ electrocatalysts, and (d) high-resolution $\mathrm{Ru} 3 \mathrm{p}$ core-level XPS spectra of $\mathrm{Pt}_{1} \mathrm{Ru}_{0.5} / \mathrm{TM}$ electrocatalysts

和 $\mathrm{Pt}_{1} \mathrm{Ru}_{0.5} / \mathrm{TM}$ 的 Pt4f XPS 谱图。 $\mathrm{Pt}_{1} \mathrm{Ru}_{0.5} / \mathrm{TM}$ 中的 $\mathrm{Pt}$ 主要以金属 $\mathrm{Pt}$ 存在, 在 71.4 和 $74.7 \mathrm{eV}$ 处出现两 个明显的金属 Pt 信号峰。对比看出, 一对 +4 价的 $\mathrm{Pt}$ 离子峰(72.9 和 $78.0 \mathrm{eV})$ 消失, +2 价的 $\mathrm{Pt}$ 离子峰 $(72.0$ 和 $76.0 \mathrm{eV})$ 的信号减弱, 说明 $\mathrm{Ru}$ 的掺入促进 $\mathrm{Pt}$ 颗粒的还原, 提高金属 $\mathrm{Pt}$ 的转化率。图 4(d)是 $\mathrm{Pt}_{1} \mathrm{Ru}_{0.5} / \mathrm{TM}$ 的 $\mathrm{Ru} 3 p$ XPS 谱图。可以看出, $\mathrm{Ru}$ 元素 主要以金属 $\mathrm{Ru}$ 和氧化物 $\mathrm{RuO}_{2}$ 存在于 $\mathrm{PtRu}$ 合金中,
$\mathrm{Ru}$ 和 $\mathrm{RuO}_{2}$ 的峰面积比约为 $2: 1$ 。即 $\mathrm{RuCl}_{3}$ 在乙二醇 的作用下被还原成金属 $\mathrm{Ru}$ 粒子，不稳定的 $\mathrm{Ru}$ 颗粒 在溶剂热法或空气中部分氧化成 $\mathrm{RuO}_{2}$, 金属氧化 物 $\mathrm{RuO}_{2}$ 的存在对于贵金属催化甲醇氧化也具有促 进作用。

\section{2 电化学测试分析}

图 5(a)是不同 $\mathrm{Ru}$ 掺量的 $\mathrm{PtRu} / \mathrm{TM}$ 催化剂在 $1 \mathrm{~mol} / \mathrm{L} \mathrm{H}_{2} \mathrm{SO}_{4}$ 中的 $\mathrm{CV}$ 曲线。电化学活性面积 

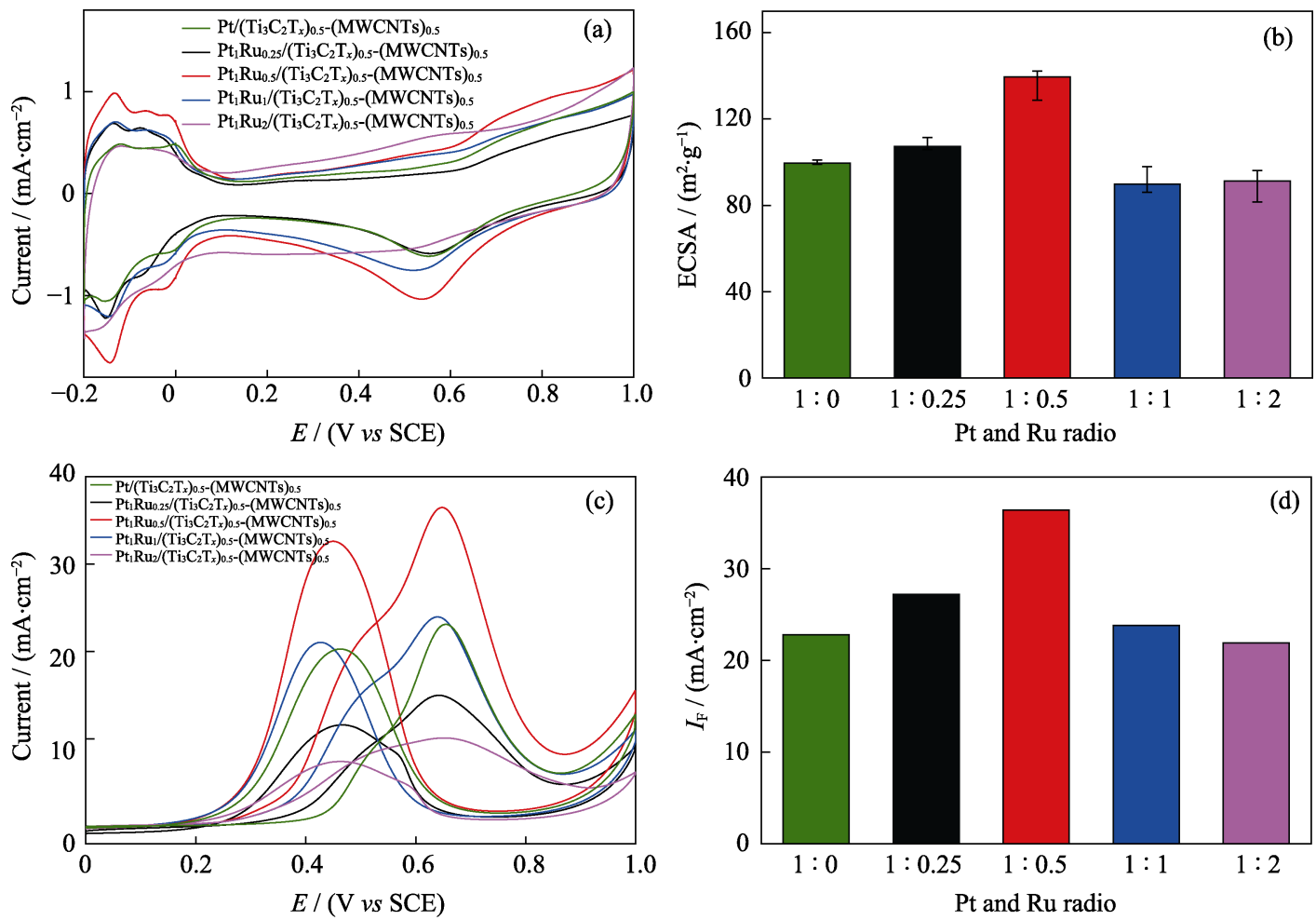

图 5 不同 $\mathrm{Ru}$ 掺量的 $\mathrm{PtRu} / \mathrm{TM}$ 在(a)1 mol/ $\mathrm{L} \mathrm{H}_{2} \mathrm{SO}_{4}$ 电解液中 $20 \mathrm{mV} / \mathrm{s}$ 扫速下的循环伏安曲线, (b)对应的 ECSA 值,

(c)在 $1 \mathrm{~mol} / \mathrm{L} \mathrm{H}_{2} \mathrm{SO}_{4}+2 \mathrm{~mol} / \mathrm{L} \mathrm{CH}_{3} \mathrm{OH}$ 电解液中 $20 \mathrm{mV} / \mathrm{s}$ 扫速下的循环伏安曲线, (d)对应的正向峰值电流密度值 $\left(I_{\mathrm{F}}\right)$

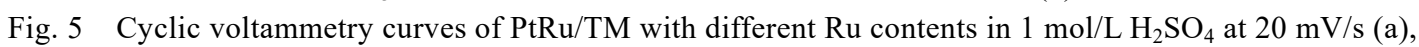
the corresponding ECSA values were shown in (b, c) in $1 \mathrm{~mol} / \mathrm{L} \mathrm{H}_{2} \mathrm{SO}_{4}+2 \mathrm{~mol} / \mathrm{L} \mathrm{CH}_{3} \mathrm{OH}$ at $20 \mathrm{mV} / \mathrm{s}$, and $(\mathrm{d})$ corresponding forward peak current densities $\left(I_{\mathrm{F}}\right)$ were shown in $(\mathrm{d})$

(Electrochemical Active Area, ECSA)是表征电催化 性能的重要指标。在-0.2 0.1 V 为析氢一脱氢区, 通

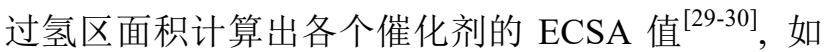
图 5(b)所示, $\mathrm{Pt} / \mathrm{TM} 、 \mathrm{Pt}_{1} \mathrm{Ru}_{0.25} / \mathrm{TM} 、 \mathrm{Pt}_{1} \mathrm{Ru}_{0.5} / \mathrm{TM}$ 、 $\mathrm{Pt}_{1} \mathrm{Ru}_{1} / \mathrm{TM}$ 和 $\mathrm{Pt}_{1} \mathrm{Ru}_{2} / \mathrm{TM}$ 的 ECSA 值分别为 99.6、 107.2、139.5、89.8 和 $91.3 \mathrm{~m}^{2} \cdot \mathrm{g}^{-1}$, 即 $\mathrm{Pt}_{1} \mathrm{Ru}_{0.5} / \mathrm{TM}$ 具 有最大的活性面积。ECSA 的值越大, 催化剂的活 性位点越多, 催化活性也越高。图 5(c) 是不同 $\mathrm{Ru}$ 掺 量的 $\mathrm{PtRu} / \mathrm{TM}$ 催化剂在 $1 \mathrm{~mol} / \mathrm{L}_{2} \mathrm{SO}_{4}+2 \mathrm{~mol} / \mathrm{L}$ $\mathrm{CH}_{3} \mathrm{OH}$ 中的循环伏安曲线, 在正向扫描 $0.7 \mathrm{~V}$ 和反 向扫描 $0.5 \mathrm{~V}$ 左右分别出现甲醇的第一次氧化峰和 中间中毒产物一氧化碳的氧化峰。如图 5(d)所示, 在正扫过程中, 不同 $\mathrm{Ru}$ 掺量的 $\mathrm{PtRu} / \mathrm{TM}$ 催化剂催 化氧化甲醇的最大电流值 $I_{\mathrm{F}}$ 分别为 $22.8 、 27.2 、 36.4$ 、 23.8 和 $21.9 \mathrm{~mA} \cdot \mathrm{cm}^{-2}, \mathrm{PtRu}$ 二元合金催化剂的 $I_{\mathrm{F}}$ 值 均大于 $\mathrm{Pt}$ 一元催化剂, 且 $\mathrm{Pt}_{1} \mathrm{Ru}_{0.5} / \mathrm{TM}$ 的 $I_{\mathrm{F}}$ 值最大, 说明该 $\mathrm{PtRu}$ 配比的电催化剂催化氧化甲醇的能力 最强, 催化剂的电催化活性最高。回扫过程中催化 氧化甲醇的峰对应的电流值记为 $I_{\mathrm{b}}, I_{\mathrm{F}} / I_{\mathrm{b}}$ 的比值用来 评价催化剂抗 $\mathrm{CO}$ 中毒能力的强弱。 $\mathrm{Pt}_{1} \mathrm{Ru}_{0.5} / \mathrm{TM}$ 的 $I_{\mathrm{F}} / I_{\mathrm{b}}$ 值为 1.13 , 大于 $\mathrm{Pt} / \mathrm{TM}$ 催化剂(1.10), 说明 $\mathrm{Pt}_{1} \mathrm{Ru}_{0.5} / \mathrm{TM}$ 催化剂抗毒化能力更强。因此适量钌
金属的加入可以提高催化剂的活性及抗 $\mathrm{CO}$ 中毒 能力。

图 6(a)是不同 $\mathrm{Ru}$ 掺量的 $\mathrm{PtRu} / \mathrm{TM}$ 催化剂的奈 奎斯特(Nyquist)图谱。Nyquist 图谱由高频区的半圆 和低频区的斜线组成。阻抗的值包括电解液阻力 $\left(R_{\mathrm{s}}\right)$ 和电荷在电极材料内部转移的阻力 $\left(R_{\mathrm{ct}}\right)$, 这两者在 图中由在高频区的半圆与实轴的交点和半圆的直径 表示。可以看出, $\mathrm{Pt}_{1} \mathrm{Ru}_{0.5} / \mathrm{TM}$ 的 $R_{\mathrm{ct}}$ 约为 $400 \Omega$, 与其 他 $\mathrm{Ru}$ 掺量相比，该掺量下 $R_{\mathrm{ct}}$ 更小。同时, $\mathrm{Pt}_{1} \mathrm{Ru}_{0.5} / \mathrm{TM}$ 复合材料在低频区的斜线近似垂直于实轴，说明其 扩散电阻较小, 导电性较好。此外, $\mathrm{Pt}_{1} \mathrm{Ru}_{0.5} / \mathrm{TM}$ 复合 材料的 $R_{\mathrm{s}}$ 值为 $1.5 \Omega$, 与 $\mathrm{Pt} / \mathrm{TM}$ 相当 $(1.29 \Omega), \mathrm{Ru}$ 的 加入有利于提高催化剂的导电性能。

通过计时电流法测试电催化剂的稳定性。图 6(b) 分别是不同 $\mathrm{Ru}$ 掺量的 $\mathrm{PtRu} / \mathrm{TM}$ 催化剂在 $1 \mathrm{~mol} / \mathrm{L}$ $\mathrm{H}_{2} \mathrm{SO}_{4}+2 \mathrm{~mol} / \mathrm{L} \mathrm{CH}_{3} \mathrm{OH}$ 中的 $I-t$ 曲线, 所有电催化 剂曲线的极化电流均随着时间的延长而逐渐衰减直 至趋于平稳。这是由于甲醇氧化形成的中间产物一 氧化碳会吸附在贵金属催化剂表面，占据活性位点， 致使催化剂中毒, 从而出现电流衰退现象 ${ }^{[31-32]}$ 。其 中, $\mathrm{Pt}_{1} \mathrm{Ru}_{0.5} / \mathrm{TM}$ 催化剂的催化电流下降最为缓慢, 说明该 $\mathrm{Ru}$ 掺量下的催化剂的稳定性最好。 

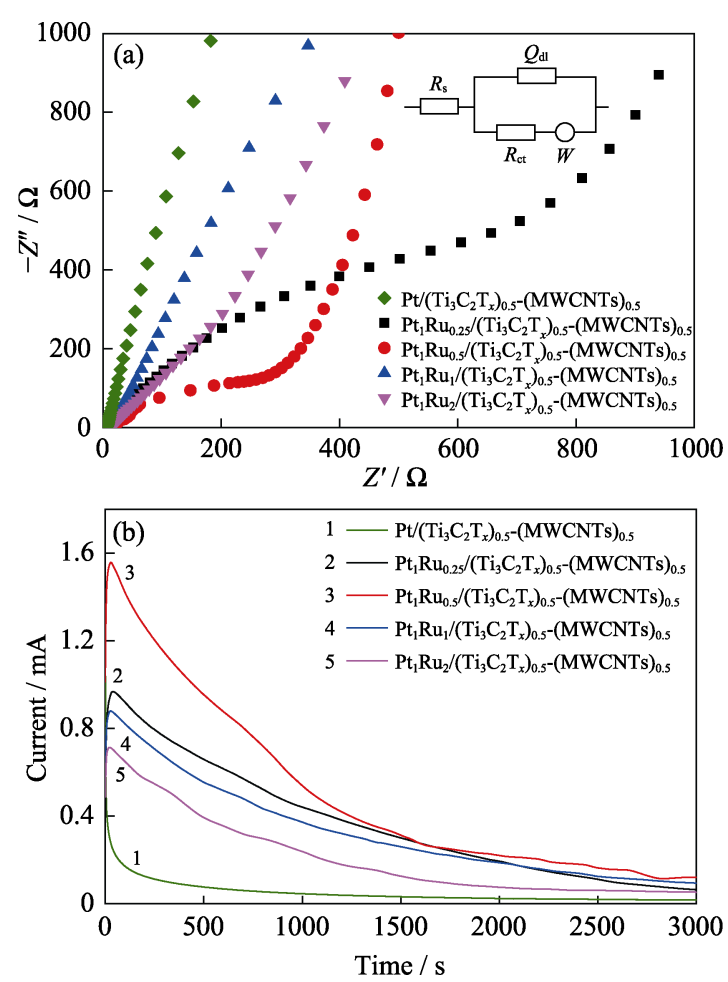

图 6 不同 $\mathrm{Ru}$ 掺量的 $\mathrm{Pt} / \mathrm{Ru} / \mathrm{TM}$ 的(a)电化学阻抗谱图和(b) 计时电流曲线

Fig. 6 (a) Nyquist plots of EIS and (b) chronoamperometric curves of $\mathrm{PtRu} /\left(\mathrm{Ti}_{3} \mathrm{C}_{2} \mathrm{~T}_{x}\right)_{0.5}$-(MWCNTs $)_{0.5}$ with different $\mathrm{Ru}$ contents in $1 \mathrm{~mol} / \mathrm{L} \mathrm{H}_{2} \mathrm{SO}_{4}+2 \mathrm{~mol} / \mathrm{L} \mathrm{CH}_{3} \mathrm{OH}$

为进一步说明 $\mathrm{Pt}_{1} \mathrm{Ru}_{0.5} / \mathrm{TM}$ 具有优异的电催化 性能, $\mathrm{PtRu} / \mathrm{TM}$ 的 $I_{\mathrm{F}} / \mathrm{ECSA}$ 与文献报道的 $\mathrm{Pt}$ 基催化 剂比较如图 7 所示。可以看到, $\mathrm{Pt}_{1} \mathrm{Ru}_{0.5} / \mathrm{TM}$ 的 $\mathrm{ECSA}$ 和 $I_{\mathrm{F}}$ 均优于 $\mathrm{Pt}$ 基催化剂 ${ }^{[33-38]}$, 说明该催化剂在燃料电 池系统中用作阳极催化剂时具有独特的优势, ECSA 值 $\left(139.5 \mathrm{~m}^{2} \cdot \mathrm{g}^{-1}\right)$ 相比 Pt/TM $\left(99.6 \mathrm{~m}^{2} \cdot \mathrm{g}^{-1}\right)$ 提高 $140 \%$, $I_{\mathrm{F}}$ 值 $\left(1285.83 \mathrm{~mA} \mathrm{mg}^{-1}\right)$ 相比 Pt/TM (805.41 $\left.\mathrm{mA} \cdot \mathrm{mg}^{-1}\right)$ 提高 $160 \%$, 表明选用 $\left(\mathrm{Ti}_{3} \mathrm{C}_{2} \mathrm{~T}_{x}\right)_{0.5}$-(MWCNTs) $)_{0.5}$ 催化 剂载体负载 PtRu 合金粒子有效提高了 $\mathrm{Pt}$ 基催化剂 的催化活性。

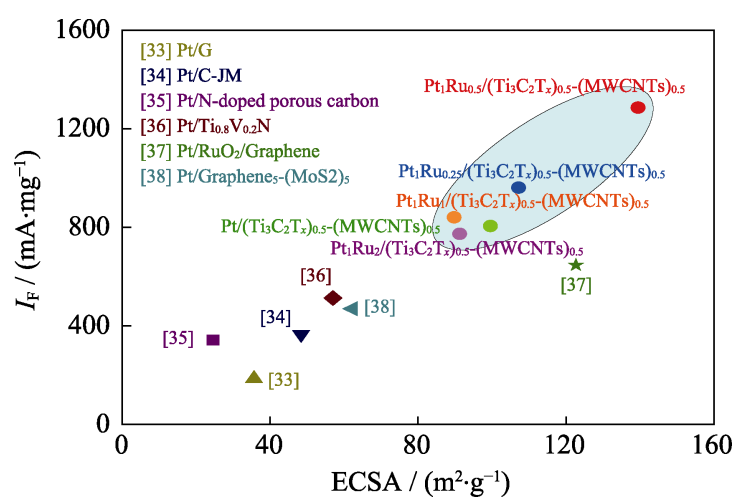

图 7 不同 $\mathrm{Ru}$ 掺量的 $\mathrm{PtRu} / \mathrm{TM}$ 与最近 $\mathrm{Pt}$ 基催化剂 $\mathrm{ECSA}$ 和 $I_{\mathrm{F}}$ 值的比较

Fig. 7 Comparison between PtRu/TM with different Ru contents and recent Pt-based catalyst of ECSA and $I_{\mathrm{F}}$ values

\section{3 结论}

以 $\mathrm{HF}$ 刻蚀 $\mathrm{Ti}_{3} \mathrm{AlC}_{2}$ 得到的 $\mathrm{Ti}_{3} \mathrm{C}_{2} \mathrm{~T}_{x}$ 复合酸化处 理的 MWCNTs，采用溶剂热法负载 Pt、Ru 粒子，制 备得到了一系列不同 $\mathrm{Pt} 、 \mathrm{Ru}$ 配比的 $\mathrm{PtRu} /\left(\mathrm{Ti}_{3} \mathrm{C}_{2} \mathrm{~T}_{x}\right)_{0.5^{-}}$ (MWCNTs) $)_{0.5}$ 催化剂。通过对催化剂的形貌和结构 进行表征后发现, PtRu/ $\left(\mathrm{Ti}_{3} \mathrm{C}_{2} \mathrm{~T}_{x}\right)_{0.5}$-(MWCNTs $)_{0.5}$ 催 化剂中 $\mathrm{Ru}$ 原子掺入 $\mathrm{Pt}$ 原子晶格中形成铂钉双金属 合金。同时，合金颗粒均匀负载在 $\mathrm{Ti}_{3} \mathrm{C}_{2} \mathrm{~T}_{x}$-MWCNTs 载体上, 大小约为 $3.6 \mathrm{~nm}$, 分散较为均匀, 有利于 催化反应活性。电化学测试发现, 与 $\mathrm{Pt} /\left(\mathrm{Ti}_{3} \mathrm{C}_{2} \mathrm{~T}_{x}\right)_{0.5}$ (MWCNTs $)_{0.5}$ 相比, PtRu/( $\left.\mathrm{Ti}_{3} \mathrm{C}_{2} \mathrm{~T}_{x}\right)_{0.5}$-(MWCNTs $)_{0.5}$ 系列催化剂表现出更好的催化活性和稳定性。其中, $\mathrm{Pt}_{1} \mathrm{Ru}_{0.5} /\left(\mathrm{Ti}_{3} \mathrm{C}_{2} \mathrm{~T}_{x}\right)_{0.5}$-(MWCNTs $)_{0.5}$ 催化剂催化甲醇 电氧化的能力和电化学稳定性均最优, 其 ECSA 为 $139.5 \mathrm{~m}^{2} / \mathrm{g}$, 正向峰电流密度为 $36.4 \mathrm{~mA} / \mathrm{cm}^{2}$, 性能 远优于其它配比的催化剂。

\section{参考文献:}

[1] CHU S, MAJUMDAR A. Opportunities and challenges for a sustainable energy future. Nature, 2012, 488(7411): 294-303.

[2] WEN Z, LIU J, LI J. Core/shell Pt/C nanoparticles embedded in mesoporous carbon as a methanol-tolerant cathode catalyst in direct methanol fuel cells. Adv. Mater., 2008, 20(4): 743-747.

[3] LIU H, SONG C, LEI Z, et al. A review of anode catalysis in the direct methanol fuel cell. J. Power Sources, 2006, 155(2): 95-110.

[4] XIAO Z, MIN Y, LIANG M, et al. Recent advances in catalysts for direct methanol fuel cells. Energy Environ. Sci., 2011, 4(8): 2736-2753.

[5] LIU M, ZHANG R, CHEN W. Graphene-supported nanoelectrocatalysts for fuel cells: synthesis, properties, and applications. Chem. Rev., 2014, 114(10): 5117-5160.

[6] LU S, EID K, GE D, et al. One-pot synthesis of PtRu nanodendrites as efficient catalysts for methanol oxidation reaction. Nanoscale, 2017, 9(3): 1033-1039.

[7] LI M, ZHENG H, HAN G, et al. Facile synthesis of binary PtRu nanoflowers for advanced electrocatalysts toward methanol oxidation. Catal. Commun., 2017, 92: 95-99.

[8] W L J, M S R, E S K, et al. How to make electrocatalysts more active for direct methanol oxidation-avoid PtRu bimetallic alloys! $J$. Phys. Chem. B, 2000, 104(42): 9772-9776.

[9] ZHANG J, CAO H, WANG H. Research progress of novel twodimensional material MXene. J. Inorg. Mater., 2017, 32(6): 561-570.

[10] ZHENG W, SUN Z, ZHANG P, et al. Research progress on MXene, two dimensional nano-materials. Mater. Rev. A, 2017, 31(9): $1-14$.

[11] YU X, YOHAN D A, MICHAEL N, et al. Prediction and characterization of MXene nanosheet anodes for non-lithium-ion batteries. ACS Nano, 2014, 8(9): 9606-9615.

[12] WEI Z, LI Y, PEIGEN Z, et al. Energy storage and application for 2D nano-material MXenes. Mater. Rev. A, 2018, 32(15): 2513-2537.

[13] YAO S, LI N, YE H, et al. Synthesis of two-dimensional MXene and their applications in electrochemical energy storage. Progress in Chemistry, 2018, 30(7): 932-946.

[14] JIANG Y, XIE X, CHEN Y, et al. Hierarchically structured cellu- 
lose aerogels with interconnected MXene networks and their enhanced microwave absorption properties. J. Mater. Chem. C, 2018, 6(32): 8679-8687.

[15] LIU Y, LUO R, LI Y, et al. Sandwich-like $\mathrm{Co}_{3} \mathrm{O}_{4} / \mathrm{MXene}$ composite with enhanced catalytic performance for bisphenol a degradation. Chem. Eng. J., 2018, 347: 731-740.

[16] ZHENG H, CHEN J, LI Y. Research on preparation and photocatalytic application of two-dimensional crystal MXene. B. Chin. Ceram. Soc., 2018, 37(06): 1908-1913.

[17] MA Y, LIU N, LI L, et al. A highly flexible and sensitive piezoresistive sensor based on MXene with greatly changed interlayer distances. Nat. Commun., 2017, 8(1): 1207-1215.

[18] HU Q K, SUN D D, WU Q H, et al. MXene: a new family of promising hydrogen storage medium. J. Phys. Chem. A, 2013, 117(51): 14253-14260.

[19] NAGUIB M, MOCHALIN V N, BARSOUM M W, et al. 25th anniversary article: MXenes: a new family of two-dimensional materials. Adv. Mater., 2014, 26(7): 992-1005.

[20] XIE X, CHEN S, DING W, et al. An extraordinarily stable catalyst: $\mathrm{Pt}$ NPs supported on two-dimensional $\mathrm{Ti}_{3} \mathrm{C}_{2} \mathrm{X}_{2} \quad(\mathrm{X}=\mathrm{OH}, \quad \mathrm{F})$ nanosheets for oxygen reduction reaction. Chem. Commun., 2013, 49(86): 10112-10114.

[21] WANG G, SUN G, QI W, et al. Effect of carbon black additive in Pt black cathode catalyst layer on direct methanol fuel cell performance. Int. J. Hydrogen Energy, 2010, 35(20): 11245-11253.

[22] YAO Z, YUE R, ZHAI C, et al. Electrochemical layer-by-layer fabrication of a novel three-dimensional Pt/graphene/carbon fiber electrode and its improved catalytic performance for methanol electrooxidation in alkaline medium. Int. J. Hydrogen Energy, 2013, 38(15): 6368-6376.

[23] KIM H T, YOU D J, YOON H K, et al. Cathode catalyst layer using supported Pt catalyst on ordered mesoporous carbon for direct methanol fuel cell. J. Power Sources, 2014, 180(2): 724-732.

[24] LI W, LIANG C, ZHOU W, et al. Preparation and characterization of multiwalled carbon nanotube-supported platinum for cathode catalysts of direct methanol fuel cells. J. Phys. Chem. B, 2003, 107(26): 149-154.

[25] ZHANG X, ZHU J X, TIWARY C S, et al. Palladium nanoparticles supported on nitrogen and sulfur dual-doped graphene as highly active electrocatalysts for formic acid and methanol oxidation. ACS Appl. Mater. Interfaces, 2016, 8(17): 10858-10865.

[26] CHEN W X, ZHAO J, LEE J Y, et al. Microwave heated polyol synthesis of carbon nanotubes supported Pt nanoparticles for methanol electrooxidation. Mater. Chem. Phys., 2005, 91(1): 124-129.

[27] WANG Z B, YIN G P, SHI P F. Effects of ozone treatment of carbon support on $\mathrm{Pt}-\mathrm{Ru} / \mathrm{C}$ catalysts performance for direct methanol fuel cell. Carbon, 2006, 44(1): 133-140.

[28] ANTONUCCI P L, ALDERUCCI V, GIORDANO N, et al. On the role of surface functional groups in Pt carbon interaction. J. Appl. Electrochem., 1994, 24(1): 58-65.

[29] WILHELMSSON O, PALMQUIST J P, LEWIN E, et al. Deposition and characterization of ternary thin films within the Ti-Al-C system by DC magnetron sputtering. J. Cryst. Growth, 2006, 291(1): 290-300.

[30] QIU J D, WANG G C, LIANG R P, et al. Controllable deposition of platinum nanoparticles on graphene as an electrocatalyst for direct methanol fuel cells. J. Phys. Chem. C, 2011, 115(31): 15639-15645.

[31] ZHANG Y, CHANG G, SHU H, et al. Synthesis of Pt-Pd bimetallic nanoparticles anchored on graphene for highly active methanol electro-oxidation. J. Power Sources, 2014, 262(262): 279-285.

[32] YANG X, YANG Q, XU J, et al. Bimetallic PtPd nanoparticles on Nafion-graphene film as catalyst for ethanol electro-oxidation. $J$. Mater. Chem., 2012, 22(16): 8057-8062.

[33] HUANG H, ZHU J, ZHANG W, et al. Controllable codoping of nitrogen and sulfur in graphene for highly efficient Li-oxygen batteries and direct methanol fuel cells. Chem. Mater, 2016, 28(6): 1737-1745.

[34] ZHANG X, ZHANG J, HUANG H, et al. Platinum nanoparticles anchored on graphene oxide-dispersed pristine carbon nanotube supports: high-performance electrocatalysts toward methanol electrooxidation. Electrochim. Acta, 2017, 258: 919-926.

[35] SU F, TIAN Z, POH C K, et al. Pt nanoparticles supported on nitrogendoped porous carbon nanospheres as an electrocatalyst for fuel cells. Chem. Mater, 2010, 22(3): 832-839.

[36] ZHANG B, PAN Z C, YU K, et al. Titanium vanadium nitride supported $\mathrm{Pt}$ nanoparticles as high-performance catalysts for methanol oxidation reaction. J. Solid State Electrochem., 2017, 21(10): 3065-3070.

[37] HUANG H, ZHU J, LI D, et al. Pt nanoparticles grown on 3D $\mathrm{RuO}_{2}$-modified graphene architectures for highly efficient methanol oxidation. J. Mater. Chem. A, 2017, 5(9): 4560-4567.

[38] GAO Z, LI M, WANG J, et al. Pt nanocrystals grown on three-dimensional architectures made from graphene and $\mathrm{MoS}_{2}$ nanosheets: highly efficient multifunctional electrocatalysts toward hydrogen evolution and methanol oxidation reactions. Carbon, 2018, 139: 369-377. 